\title{
Biomechanical Characterization of Intracranial Aneurysm Wall: A Multiscale Study
}

\author{
Francesco Signorelli ${ }^{1,2}$, Cyril Pailler-Mattei ${ }^{6,7}$, Benjamin Gory $^{8}$, Pierre Larquet $^{7}$, Philip Robinson $^{3}$, Roberto Vargiolu $^{6}$, \\ Hassan Zahouani ${ }^{6}$, Paul-Emile Labeyrie ${ }^{4}$, Jacques Guyotat ${ }^{2}$, Isabelle Pelissou-Guyotat ${ }^{2}$, Julien Berthiller ${ }^{5}$, \\ Francis Turjman ${ }^{4}$
}

- OBJECTIVE: Aneurysm wall biomechanics are not yet an integral part of aneurysm rupture risk evaluation. We aimed to develop a new technique describing the biomechanical properties of aneurysm wall and correlating them to rupture status.

- METHODS: Aneurysm wall samples collected during surgery were submitted before and after freezing to tensile tests or as fresh samples to indentation tests. The lateral stiffness or the Young's modulus of the different samples was determined as a function of the mechanical test used. The impact of freezing on biomechanical properties was evaluated. The correlation of clinical and radiologic data with the biomechanical profile of the aneurysm samples was investigated. Two-photon microscopy was used to study collagen fiber organization.

- RESULTS: Sixteen aneurysm samples (11 unruptured and 5 ruptured) were included. Freezing decreased tissue stiffness. No significant difference was found between ruptured and unruptured aneurysm wall samples regarding demographic characteristics, ethnicity, smoking status, arterial hypertension, site, size and shape of the aneurysm, PHASES score, mechanical profile, or overall Young's modulus. Indentation tests found that the rupture occurred in a restricted area of increased elastic capacity and unruptured areas had increased stiffness. Two-photon microscopy found disruption of the collagen fiber network in rupture zones.

\footnotetext{
Key words

- Biomechanics

- Intracranial aneurysm

- Rupture

- Young's modulus
}

Abbreviations and Acronyms RIA

Ruptured intracranial aneurysm TPM

2-Photon microscopy

UIA: Unruptured intracranial aneurysm

From the ${ }^{1}$ Division of Neurosurgery, Department of Basic Medical Sciences, Neurosciences and Sense Organs, University "Aldo Moro" of Bari, Bari, Italy; ${ }^{2}$ Division of Neurosurgery,

${ }^{3}$ Direction of Clinical Research and Innovation, ${ }^{4}$ Department of Interventional
- CONCLUSIONS: The indentation test of fresh aneurysm wall samples described the heterogeneity of biomechanical properties of the tissue and found increased elastic capacity in the rupture zone and increased stiffness in the remainder of the aneurysm. This study could be a basis for further research aimed at building a biomechanical-based model of aneurysm rupture risk.

\section{INTRODUCTION}

$\mathrm{P}$ rognosis of aneurysmal rupture remains dismal, notwithstanding continuous progress in its medical and surgical management ${ }^{\mathrm{I}-4}$ and preventing this event is desirable. However, clinicians and patients are often confronted with the dilemma as to whether or not to treat unruptured intracranial aneurysms (UIAs) because individualized prediction of rupture risk is not certain and the risks associated with treatment remain high. ${ }^{-8}$

The methods currently used to predict risk of rupture are based essentially on demographics, clinical data, and aneurysm location and morphology. ${ }^{2,5,8}$ However, as the aneurysm wall ruptures when the transmural pressure exceeds its tensile strength, ${ }^{9, \text { IO }}$ a method of predicting the risk of rupture based on the evaluation of the mechanical properties of the aneurysm wall could prove more reliable. There have been attempts ${ }^{4, I I, I 2}$ to address this (e.g., studies using computational fluid dynamics set out to find a correlation between hemodynamic factors [wall shear stress, wall

Neuroradiology, and ${ }^{\mathbf{5}}$ Unit of methodological support, "Pôle Information Médicale Evaluation Recherche", Hospices Civils de Lyon, Lyon; ${ }^{6}$ Tribology and Systems Dynamics Laboratory, UMR-CNRS 5513, "Ecole Centrale de Lyon", Ecully, "University "Claude Bernard Lyon 1", ISPB-Faculty of Pharmacy, Lyon, and ${ }^{\mathbf{8}}$ Department of Diagnostic and Interventional Neuroradiology, University Hospital of Nancy, Nancy, France

To whom correspondence should be addressed: Francesco Signorelli, M.D., M.Sc. [E-mail: francesco.signorelli@uniba.it] 
shear stress gradient, oscillatory shear index, intra-aneurysmal flow patterns] and structural changes of aneurysm wall predisposing to rupture). However, most studies ${ }^{4, I 1, I 2}$ are retrospective, did not consider wall pulsatility, did not measure patient-specific flow rates, and there is conflicting evidence about what should be considered a precursor to rupture. In addition, accurate data regarding the structural and biomechanical alterations of the aneurysm wall inducing its rupture is still lacking. Current techniques to evaluate aneurysm wall biomechanics are based on tensile testing. ${ }^{\mathrm{IO}, \mathrm{I3}}$ These techniques test aneurysm wall samples after freezing, owing to logistic difficulties of testing fresh samples, ${ }^{\text {IO }}$ which may alter biological tissue biomechanics, but this has yet to be formally investigated and is the subject of the present report. In addition, tensile testing estimates the biomechanical properties of the aneurysm as a whole or with a low or unspecified spatial resolution, ${ }^{\mathrm{IO}, \mathrm{I} 3}$ whereas clinical evidence suggests that alterations of aneurysm wall at the rupture site is generally confined to a restricted zone of the fundus. ${ }^{\text {I4 }}$ Thus, a local technique for studying the biomechanical properties of the aneurysm wall ex vivo with a high spatial resolution, considering the anisotropy and heterogeneity of the aneurysm wall structure, was developed and is reported herein.

\section{METHODS}

\section{Population}

All patients who underwent surgery for an intracranial aneurysm between December 2013 and March 2014 were prospectively identified. Only those having provided written informed consent were included. Patients whose aneurysm wall sample was too small ( $<5 \mathrm{~mm}$ in the major axis) to be placed between the grips of the traction device (that were set to a start distance of $3 \mathrm{~mm}$, corresponding to the initial, minimal, distance between the 2 fastenings necessary for the tensile test) or, for the indentation test, $<2 \mathrm{~mm}^{2}$ and examined after $\mathrm{I} 8$ hours of sampling, were excluded. The I8-hour threshold was chosen, as in our opinion, it represented the best compromise between technical constraints entailed by our biomechanical analysis, that required $\geq_{\mathrm{I} 2}$ hours, and risk of artifacts associated with tissue lysis, which is present after 24 hours. ${ }^{15}$ This study was approved by the local ethics committee.

\section{Clinical and Radiologic Data Collection}

For each patient included we prospectively collected data regarding demographics (sex, ethnic origin, age), acquired risk factors for rupture (cigarette smoking, alcohol consumption, arterial hypertension), familial aneurysms, ruptured versus unruptured status, aneurysm size and shape (regular vs. irregular), site, and previous history of aneurysmal subarachnoid hemorrhage. PHASES score ${ }^{5}$ was calculated by a neurosurgeon familiar with the evaluation of patients with aneurysmal subarachnoid hemorrhage. All aneurysm features were determined by a neuroradiologist blinded to the ruptured/unruptured state on a preoperative brain digital subtraction angiography.

\section{Sampling and Preparation of Aneurysmal Wall Samples}

After surgical clipping, the aneurysm wall was collected by cutting it as much as possible parallel and inferior to the equator of the aneurysm, aiming at including the fundus as well as the region next to the neck. The part of the aneurysm proximal to the clip was marked with methylene blue to match the intraoperative photos of the aneurysm with the images of the aneurysm during the biomechanical tests and 2-photon microscopy before and after freezing. Each aneurysm sample was immersed in $0.9 \%$ saline solution immediately after sampling, stored in an insulated cooler, and analyzed within 18 hours of sampling. Two types of investigation were performed - an indentation test to obtain a mechanical cartography of the aneurysm wall, and a uniaxial tensile test to evaluate the effects of freezing (performed before and after freezing). For the latter, the freezing procedure consisted of placing aneurysm wall samples directly in a freezer, after a protecting treatment for 20 minutes at $+4{ }^{\circ} \mathrm{C}$ with $15 \%$ dimethylsulfoxide, where they reached the temperature of $-80^{\circ} \mathrm{C}$ in 2 hours. $^{\text {Io, } 15}$ The frozen samples were stored for a minimum I month, then thawed $\left(+40^{\circ} \mathrm{C}\right.$ water bath for ro minutes then maintained at $+4{ }^{\circ} \mathrm{C}$ until completely thawed) for no longer than 24 hours before biomechanical testing. ${ }^{\text {IO,I5 }}$ All samples (fresh and frozen) were placed at room temperature $\left(+24^{\circ} \mathrm{C}\right)$ for 30 minutes before biomechanical testing. Unloaded wall thickness was measured at 5 positions on each sample using microcalipers and averaged. ${ }^{\mathrm{I}}$

\section{Mechanical Properties of Aneurysm Wall}

Each sample was submitted to tensile or indentation tests in its entirety. The lateral stiffness or the Young's modulus of the different samples were determined as function of the mechanical test used. All mechanical tests were performed at room temperature $\left(24^{\circ} \mathrm{C}\right)$.

Uniaxial Tensile Test. The uniaxial tensile test consists in recording the elongation (strain) of the sample tested as a function of the force applied. This method is commonly used to estimate the strength of the aneurysm wall to mechanical deformation. ${ }^{\text {Io, } 13,15}$ The aneurysm samples were inserted between 2 specific fasten-ings $(3 \times 3 \times 5 \mathrm{~mm})$, which firmly clamped the aneurysm to avoid the sample microsliding during the tensile tests. The initial dis-tance between the 2 fastenings was $3 \mathrm{~mm}$. All along the uniaxial tensile test, the aneurysms were continuously irrigated with saline to avoid dehydration, which could affect the reproducibility of the mechanical measurements. The uniaxial test was carried out in controlled displacement mode. The $x$-displacement (distance between the 2 fastenings induced by the displacement table from the initial position of the 2 fastenings) was obtained from the displacement table (PI-629-ICD, National Instrument, Austin, Texas, USA) and controlled by a displacement sensor (Figure 1A). The uniaxial tensile tests were performed for a constant displacement (relative stretch of the aneurysm sample induced by the displacement table between the 2 fastenings) of I $\mathrm{mm}$ and a constant tensile velocity of $0.5 \mathrm{~mm} / \mathrm{s}$ to minimize the viscoelastic behavior, more precisely the stress relaxation, or creep, of the sample during the tensile test. ${ }^{\text {I }}$ A controlled prestress (Io $\mathrm{mN}$ ) was applied on each aneurysm sample to obtain the same initial conditions before uniaxial tensile test. The uniaxial tensile test allows for the evaluation of the average mechanical parameters of the aneurysm sample in the $x$-direction, such as the lateral stiffness, $k_{x}$. The latter is the 

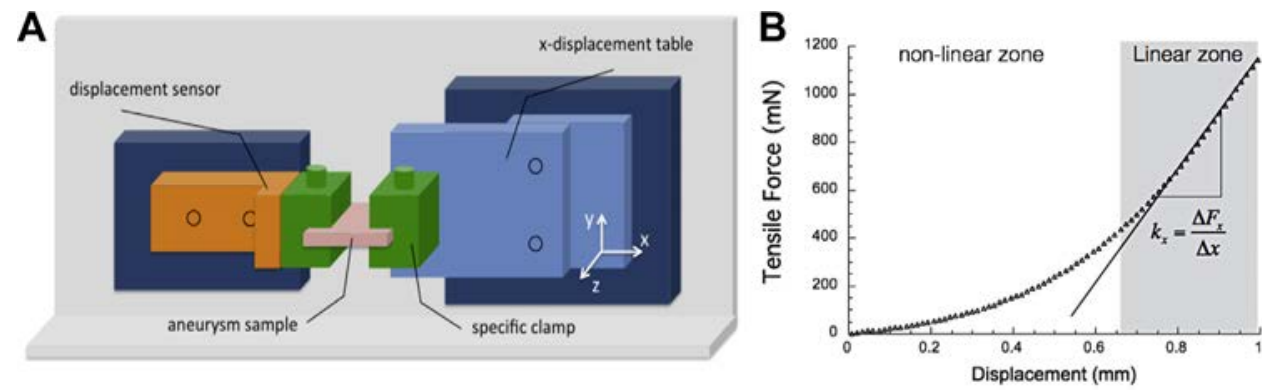

Figure 1. (A) Schematic representation of the uniaxial tensile test used to evaluate the aneurysm mechanical

properties in the $\mathrm{x}$-direction. (B) Representation of an aneurysm tensile curve obtained before freezing.

slope of the linear part of the tensile curve (Figure 1B), defined as: $k_{x}=\frac{\Delta F_{x}}{\Delta x}$, where $\Delta F_{x}$ is the variation of the tensile force and $\Delta x$ is the variation of the lateral displacement in the linear part of the curve. Also, the characteristic time, defined as the ratio between the length of the nonlinear zone and the tensile speed, which corresponds to the time necessary for the biological sample to reach the linear mechanical behavior (linear zone), was calculated before and after freezing.

The tension applied to each aneurysm sample was always below the critical load, defined as the load beyond which the material could undergo plastic deformation, to avoid permanent deformation and alteration.

Indentation Test. A light load indentation device, previously described in the literature, ${ }^{16}$ was developed to study the mechanical properties of the biological tissues (Figure 2A).

The indentation test consists of recording the penetration depth, $\delta_{y}$, of a rigid indenter as a function of applied normal load, $\mathrm{F}_{y}$, during loading/unloading experiments. A representative indentation curve for an aneurysm sample is reported in Figure 2B. The indentation tests were performed in controlled displacement mode. The $y$-displacement is obtained by the displacement table (PI-629-ICD, National Instrument) and controlled by a displacement sensor. The maximum $y$-displacement during the loading/unloading cycle can reach $500 \mu \mathrm{m}$ with a spatial resolution of $\mathrm{I} \mu \mathrm{m}$. The experimental device offers a wide range of indenting velocities from $\mathrm{I}-\mathrm{IOO} \mu \mathrm{m} / \mathrm{s}$. The experimental tests were performed for constant applied normal load $F_{y}=\mathrm{I} \mathrm{mN}$ and constant indentation speed $V=$ Io $\mu \mathrm{m} / \mathrm{s}$. A spherical steel indenter was used (radius of curvature, $R=0.5 \mathrm{~mm}$ ). The aneurysm samples were embedded into an inert natural polymer gel (alginate) for testing. The indentation tests were performed in total immersion in saline to maintain the humidity of the aneurysm samples constant and to avoid the adhesion effect between the probe and the biological tissue. ${ }^{17}$ The indentation test allows the determination of the reduced Young's modulus $\left(E_{y}^{\star}\right)$ of the aneurysm wall in the $y$-direction, from the measurement of the normal contact stiffness in the $y$-direction $\left(k_{y}\right)$, which is the slope of the initial portion of the unloading curve (Figure 2B). ${ }^{\mathrm{I}}{ }^{2}$ The contact geometry between the indenter and the material was considered for the calculation of the Young's modulus: the nature of the contact geometry was integrated into the calculation of the normal projected contact area between the aneurysm and the indenter using Hertzian

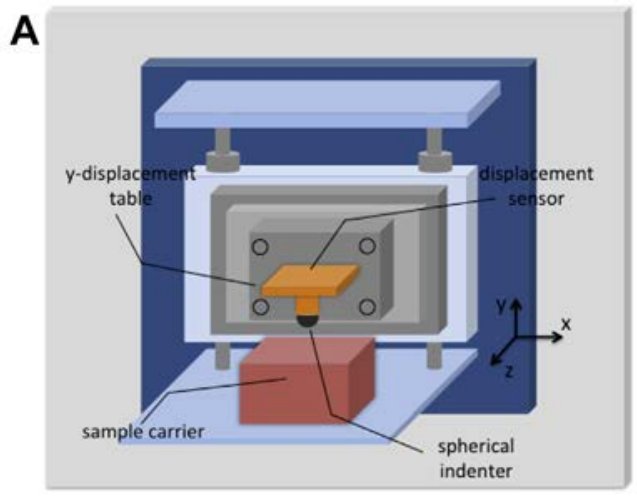

Figure 2. (A) Schematic representation of the indentation device used to evaluate the aneurysm mechanical properties in the $y$-direction. (B)
B

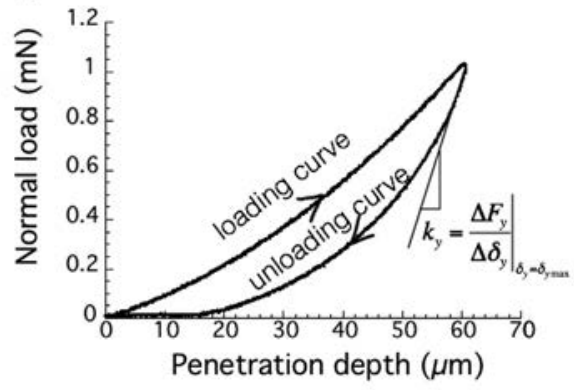

Characteristic indentation curve obtained for the aneurysm wall. 
theory. For a bulk material in contact with an axisymmetric rigid indenter, the normal stiffness is related to the $E_{y}^{\star}$ by ${ }^{19}: E_{y}^{\star}=$ $\frac{\sqrt{\pi}}{2} \frac{k_{y}}{\sqrt{A}}$, where $A$ is the normal projected contact area (in the $y$ direction) between the aneurysm and the indenter. ${ }^{18}$ The polymeric substrate effect was considered estimating the aneurysm $E_{y}^{\star}{ }^{16}$ Indentation was plotted as a force-displacement curve. $^{20,2 I}$ Stress-strain curves could not be reliably used for indentation test mainly because it was extremely difficult to estimate precisely the strain applied to the material by the indenter and because the real contact area between the indenter and the material, which depends on the contact geometry, mechanical behavior of the material, and adhesion forces between the indenter and the material was difficult to estimate.

To take advantage of the high spatial resolution of the technique and to obtain a detailed mechanical mapping of the aneurysmal wall, the surface of each sample was divided in squares of $\mathrm{I} \mathrm{mm}^{2}$, which for all samples corresponded to the approximate dimension of the aneurysm rupture zone, defined as the region of the aneurysm wall that under operating microscope magnification showed a thrombus very adherent to its exoluminal surface. ${ }^{\text {I4 }}$ A mean $E_{y}^{\star}$ was thus calculated for each $\mathrm{I}-\mathrm{mm}^{2}$ zone and recorded. In addition, each ruptured or unruptured aneurysm sample was assigned to I of 3 groups of different mechanical profile, defined as a function of their average reduced Young's modulus value $\left(E_{y}^{\star}\right.$ mean $)$, expressed by: $\frac{I}{n} \sum_{i=I}^{n} E_{y i}^{\star}$, where $n$ is the total number of measurements on the aneurysm wall sample and $E_{y i}^{\star}$ is the ith value of the measured Young's modulus: rigid profile: $\mathrm{E}_{\mathrm{y} \text { mean }}^{\star} \geq \mathrm{I} 2 \mathrm{O} \mathrm{kPa}$; intermediate profile: $8 \mathrm{o} \mathrm{kPa} \leq \mathrm{E}_{\mathrm{y} \text { mean }}^{\star}<\mathrm{I} 2 \mathrm{O}$ $\mathrm{kPa}$; elastic profile: $\mathrm{E}_{\mathrm{y} \text { mean }}^{\star}<8 \mathrm{o} \mathrm{kPa}$.

\section{Statistics}

Categorical variables were expressed as number (n) and percentage and quantitative variables were expressed as median with first and third quartiles (QI-Q3). Due to the small sample size, categorical variables were compared using Fisher's exact test and Wilcoxon nonparametric test was applied to compare the $E_{y}^{\star}$ mean of the rupture zone with the one of the intact zones of ruptured aneurysms and the $E_{y}^{\star}$ mean of intact regions of UIA and ruptured intracranial aneurysms (RIAs). Statistical tests were bilateral and the level of significance was set to $5 \%(\mathrm{P}<0.05)$. Statistical analyses were conducted using SAS version 9.3 (SAS Institute Inc., Cary, North Carolina, USA).

\section{Two-Photon Microscopy}

Two-photon microscopy (TPM), a 3-dimensional fluorescence microscopic technique based on 2-photon excitation of fluorophores, ${ }^{22}$ is a powerful method to study the 3 -dimensional distribution of molecules deep inside living tissue. In the present study, TPM was used to observe collagen fibers (excitation wavelength used $800 \mathrm{~nm}$ within the fresh aneurysm wall samples and intrinsic contrasts such as the second harmonic generation).

\section{RESULTS}

\section{Patient and Aneurysm Characteristics}

Between December 2013 and March 2014, 27 aneurysms from 27 patients were surgically repaired. Four aneurysms were excluded for reason of sample size, and a further 7 were excluded because the aneurysm was not tested within I8 hours of collection. A total of I6 samples of aneurysm wall were included (II UIAs and 5 RIAs).

\section{Impact of Freezing on Biomechanical Properties of Aneurysm Wall}

Three UIA wall samples were analyzed by tensile tests (Table 1). The small number of samples allowed only relative comparison of lateral stiffness values and graphic comparison of the curves corresponding to their biomechanical properties before and after freezing.

The mechanical behavior of the aneurysm samples was always in the linear zone, confirming that the load applied did not alter their elastic properties. Tensile tests found a 2.6- to 4.9-fold decrease of aneurysm wall stiffness after freezing (Table 1).

Also, the characteristic time significantly decreased after freezing (Figure 3A). Before freezing the mean (standard deviation) characteristic time for the 3 samples was I.36 ( \pm 0.05$)$ seconds, whereas it was $0.48( \pm 0.05)$ seconds after freezing.

TPM examination of UIAs after freezing showed a loss and disruption of collagen fiber architecture at the luminal side compared with fresh samples (Figure 3B and C).

\section{Biomechanical Properties of Fresh Aneurysmal Wall Samples}

Thirteen samples ( 8 unruptured and 5 ruptured) were analyzed by indentation tests (Table 2).

There were 6 women $(46 . \mathrm{I} \%)$ and the median age (QI-Q3) was 46 years (range, $40-56$ years); $38.5 \%$ of patients were current smokers and $69.2 \%$ were medically treated for arterial hypertension. Most aneurysms (6/13, 4 unruptured and 2 ruptured) were located at the middle cerebral artery bifurcation, 4 of 13 (2 unruptured and 2 ruptured) at the anterior communicating artery complex, 2 of 13 (I ruptured and I unruptured), at the Mi segment of the middle cerebral artery, and I at internal carotid artery

\begin{tabular}{|c|c|c|c|c|c|c|c|c|c|}
\hline ID & Age (Years) & Sex & Smoker & Status & PHASES Score & Site & Size (mm) & $k_{x}(N / m)$ Before Freezing & $k_{x}(N / m)$ After Freezing \\
\hline 1 & 33 & M & Yes & U & 8 & MCA & 18 & 1785 & 365 \\
\hline 2 & 45 & $\mathrm{~F}$ & No & U & 8 & MCA & 18 & 2130 & 595 \\
\hline 3 & 40 & M & Yes & U & 13 & MCA & 25 & 2190 & 825 \\
\hline
\end{tabular}

$\mathrm{k}_{\mathrm{x}}$, average lateral stiffness; $\mathrm{M}$, male; $\mathrm{U}$, unruptured; MCA, middle cerebral artery bifurcation; $F$, female 

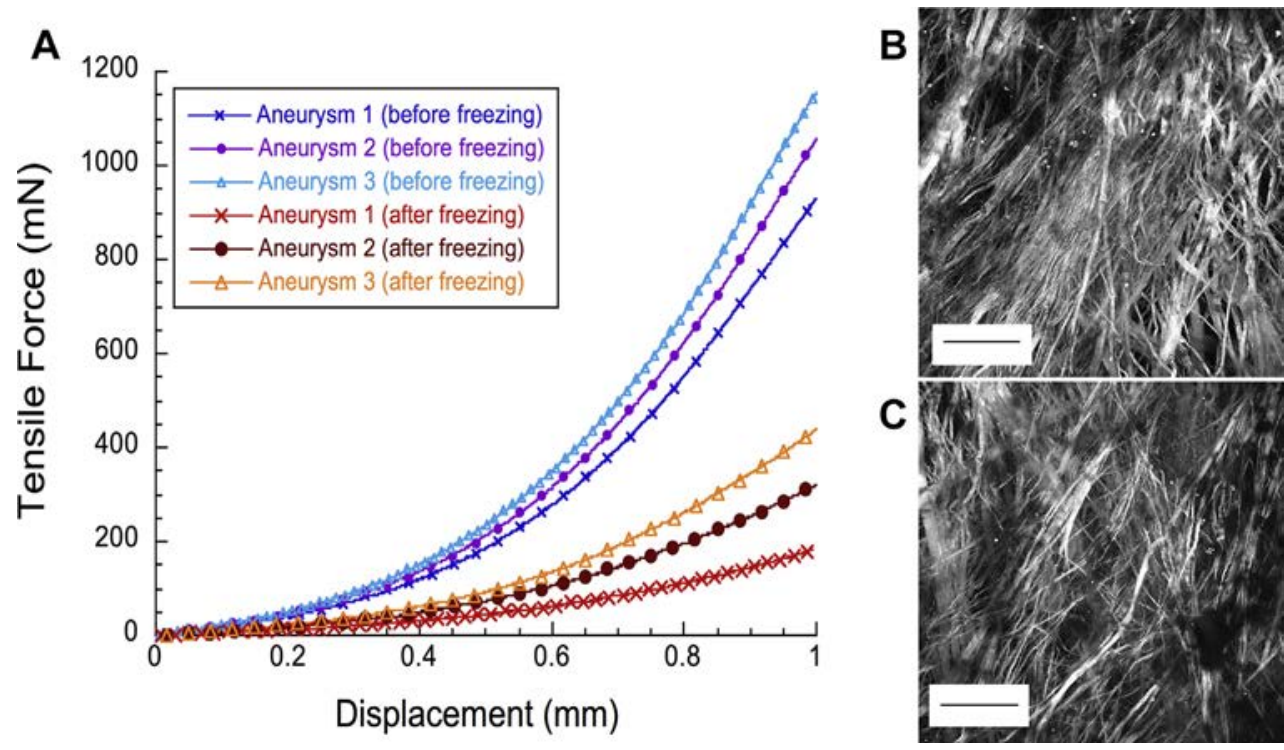

Figure 3. (A) Tensile curves for 3 unruptured aneurysm wall samples before and after freezing. Frontal sections of 1 of these aneurysm wall specimens acquired with 2-photon microscopy before $(\mathbf{B})$ and after $(\mathbf{C})$ freezing.

bifurcation (unruptured). Eight aneurysms (5 unruptured and 3 ruptured) had irregular shape and 5 ( 3 unruptured and 2 ruptured) had regular shape. The median size (QI-Q3) of the 13 aneurysms in the major axis was io $\mathrm{mm}$ (range, 7- $15 \mathrm{~mm}$ ). It was $10.5 \mathrm{~mm}$ (range, $7.5^{-}-\mathrm{r} 6.5 \mathrm{~mm}$ ) for unruptured and $8 \mathrm{~mm}$ (range, $5^{-\mathrm{II}} \mathrm{mm}$ ) for ruptured aneurysms. The median length (major axis) of the aneurysm samples was Io $\mathrm{mm}$, and their median width (calculated as the largest sample dimension perpendicular to the major axis) was $2 \mathrm{~mm}$. Wall thickness was not homogeneous. Its median value (QI-Q3) was $5 \mathrm{I} 5 \mu \mathrm{m}(273.5-6 \mathrm{I} 4 \mu \mathrm{m})$. Median (QI-Q3) PHASES score was 9 (range, 6-Io) for UIAs and 6 (range, 5-8) for RIAs. The most frequent mechanical profile was elastic $(46.2 \% ; 4$ unruptured and 2 ruptured), followed by rigid (30.8\%; 2 unruptured and 2 ruptured), and intermediate (23.1\%; 2 unruptured and I ruptured; Table 3). There was no significant difference between UIA and RIA wall samples with regard to demographics, ethnicity, smoking status, arterial hypertension, site, size and shape of the aneurysm, PHASES score, mechanical profile, or overall $E_{y \text { mean }}^{\star}$ (Table 3). Overall, $333 \mathrm{I}-\mathrm{mm}^{2}$ zones were examined by indentation tests, 205 for UIAs and 128 for RIAs. The mapping of the $E_{y}^{\star}$ for each sample was visualized by a color-coded map that represents aneurysm wall mechanical heterogeneity (Figure 4A and B). In all 5 samples of RIAs the rupture zone corresponded to one square of the superposed grid, whereas the intact region corresponded to a mean 24.6 squares (range, I8-42 squares).

The $E_{y \text { mean }}^{\star}$ was significantly higher in the intact regions of RIA (II9.8 $\mathrm{kPa}$ ) compared witho the rupture zone $(57.6 \mathrm{kPa} ; \mathrm{P}<$ o.ooor). We also compared the $\mathrm{E}_{y \text { mean }}^{\star}$ of intact zones of UIAs and RIAs and found that the intact zones of UIAs had a statistically significant higher elastic capacity (Io8.I kPa vs. IIg.8 kPa; P = o.02). Two-photon microscopy examination of wall samples of
Images were acquired on the luminal side at the same region of the aneurysm fundus, $10 \mu \mathrm{m}$ below the surface, and shows a loss and disruption of collagen fibers after freezing. Scale bars, $50 \mu \mathrm{m}$.

RIAs showed fragmentation, rarefaction, and loss of network organization of collagen fibers at the rupture zone on the luminal side (Figure 4C and D).

\section{DISCUSSION}

The present study found that the indentation test of fresh aneurysm wall samples reliably described the heterogeneity of biomechanical properties of the tissue, demonstrating that rupture occurred in a restricted area of increased elastic capacity.

In preliminary tests it was found that freezing decreased aneurysm wall stiffness, as indicated by the decreased characteristic time of frozen aneurysm wall samples compared with fresh ones. Considering that the characteristic time is the time needed for most collagen fibers to be orientated in the tensile direction and that it is strongly associated with the length and number of collagen fibers per volume unit, ${ }^{\mathrm{I}}$ loss and disruption of collagen fiber architecture after freezing, as found by 2-photon microscopy, may account for the loss of stiffness after freezing. Thus, the evaluation of patientspecific biomechanical properties of the aneurysm wall is more reliable if performed on fresh tissue samples.

Contrary to what was reported by other investigators who used tensile tests, ${ }^{\text {TO }}$ no significant difference of biomechanical properties of the whole sample was found between RIAs and UIAs in indentation tests. In the present study, this finding could be related to the use of fresh samples, which did not exhibit the fragmentation and disorganization of collagen fibers we found to be associated with freezing. However, the objective of our study was not to correlate the overall biomechanical properties of aneurysm samples with the ruptured or unruptured status, which could be the object of further research. We 
Table 2. Characteristics of 13 Patients and Aneurysms Analyzed by Indentation Tests

\begin{tabular}{|c|c|c|c|}
\hline & $n(\%)$ & Median (01-03) & Range \\
\hline Age (years) & 13 & $46(40-56)$ & $33-60$ \\
\hline Female sex & $6(46.1)$ & & \\
\hline \multicolumn{4}{|l|}{ Ethnicity } \\
\hline Caucasian & $11(84.6)$ & & \\
\hline Asiatic & $1(7.7)$ & & \\
\hline North African & $1(7.7)$ & & \\
\hline Height (cm) & 13 & $170(165-178)$ & 149-190 \\
\hline Weight (kg) & 13 & 75 (56-88) & $45-100$ \\
\hline Cigarette smoke & $5(38.5)$ & & \\
\hline Hypertension & $9(69.2)$ & & \\
\hline \multicolumn{4}{|l|}{ Aneurysm site } \\
\hline $\mathrm{ACoA}$ & $4(30.8)$ & & \\
\hline MCA bifurcation & $6(46.1)$ & & \\
\hline M1 & $2(15.4)$ & & \\
\hline ICA & $1(7.7)$ & & \\
\hline \multicolumn{4}{|l|}{ Aneurysm status } \\
\hline Ruptured & $8(61.5)$ & & \\
\hline Unruptured & $5(38.5)$ & & \\
\hline \multicolumn{4}{|l|}{ Aneurysm shape } \\
\hline Regular & $5(38.5)$ & & \\
\hline Irregular & $8(61.5)$ & & \\
\hline Aneurysm size (mm) & 13 & $10(7-15)$ & $4-25$ \\
\hline Phases score & 13 & $8(5-9)$ & $4-13$ \\
\hline \multicolumn{4}{|l|}{ Wall profile } \\
\hline Rigid & $4(30.8)$ & & \\
\hline Intermediate & $3(23.1)$ & & \\
\hline Elastic & $6(46.2)$ & & \\
\hline
\end{tabular}

Min, minimum; Max, maximum; $\mathrm{ACoA}$, anterior communicating artery; MCA, middle cerebral artery; ICA, internal carotid artery.

developed a new technique based on indentation for exploring the biomechanical heterogeneity of the aneurysm wall, finding a wide variation of stiffness for different areas of a given aneurysm. Current techniques that apply tension stress only measure the average behavior of the tissue and apply a hyperelastic model with supraphysiologic mechanical solicitations. ${ }^{10,13,23}$ In addition, the sample size of our study and that of other investigators ${ }^{10,13,23}$ is a limiting factor for tensile test, which does not allow a reliable biaxial assay. Therefore these techniques are inadequate to describe the heterogeneity of the stiffness of the aneurysm wall that depends on its viscoelastic properties, ${ }^{24}$ and do not accurately characterize UIAs and RIAs. This is the first study that applies the indentation technique to obtain a map of the
Table 3. Comparison of Features Between 8 Unruptured and 5 Ruptured Intracranial Aneurysms Whose Sample Was Analyzed by Indentation Tests

\begin{tabular}{|c|c|c|c|}
\hline & UIAs $(n=8)$ & RIAs $(n=5)$ & $P$ Value \\
\hline Age (years) & $49.5(40.0-58.0)$ & $46(40-50)$ & $0.51^{*}$ \\
\hline Sex & & & $0.99^{\dagger}$ \\
\hline Females & $4(50.0)$ & $2(40.0)$ & \\
\hline Males & $4(50.0)$ & $3(60.0)$ & \\
\hline Height (cm) & $171.0(160.0-181.5)$ & $170(170-170)$ & $0.72^{*}$ \\
\hline Weight $(\mathrm{kg})$ & $74(53-90)$ & $77(60-80)$ & $0.51^{*}$ \\
\hline \multicolumn{2}{|c|}{ Smoking status (current) } & & $0.56^{\dagger}$ \\
\hline No & $4(50.0)$ & $4(80.0)$ & \\
\hline Yes & $4(50.0)$ & $1(20.0)$ & \\
\hline Hypertension & & & $0.99^{\dagger}$ \\
\hline No & $2(25.0)$ & $2(40.0)$ & \\
\hline Yes & $6(75.0)$ & $3(60.0)$ & \\
\hline Site & & & $0.99^{\dagger}$ \\
\hline $\mathrm{ACoA}$ & $2(25.0)$ & $2(40.0)$ & \\
\hline MCA bifurcation & $4(50.0)$ & $2(40.0)$ & \\
\hline M1 & $1(12.5)$ & $1(20.0)$ & \\
\hline ICA & $1(12.5)$ & $0(0.0)$ & \\
\hline Shape & & & $0.99^{\dagger}$ \\
\hline Regular & $3(37.5)$ & $2(40.0)$ & \\
\hline Irregular & $5(62.5)$ & $3(60.0)$ & \\
\hline Size $(\mathrm{mm})$ & $10.5(7.5-16.5)$ & $8(5-11)$ & $0.51^{*}$ \\
\hline Phases score & $9(6-10)$ & $6(5-8)$ & $0.23^{*}$ \\
\hline Profile & & & $0.99^{\dagger}$ \\
\hline Rigid & $2(25.0)$ & $2(40.0)$ & \\
\hline Intermediate & $2(25.0)$ & $1(20.0)$ & \\
\hline Elastic & $4(50.0)$ & $2(40.0)$ & \\
\hline Overall $E_{y}^{\star}$ & $95.0(75.0-137.5)$ & $102.9(77.7-123.7)$ & $0.71^{*}$ \\
\hline
\end{tabular}

Data presented are either $n(\%)$ or median (01-03).

UIAs, unruptured intracranial aneurysms; RIAs, ruptured intracranial aneurysms; $A C O A$, anterior communicating artery; MCA, middle cerebral artery; ICA, internal carotid artery; $E_{y}^{\star}$, mean Young's modulus.

*Wilcoxon non-parametric test.

†Fisher exact test.

Young's modulus of intracranial aneurysm wall, its spatial resolution being of I mm, much more than the tensile test, which extracts an average value for the whole sample..$^{10,16,25}$ In the present study, rupture zones had a lower Young's modulus, thus an increased elastic capacity, than unruptured zones. This finding indicates a difference in the biomechanical properties between RIAs and UIAs at the millimetric scale, yet they have the same biomechanical profile if taken as a whole. 

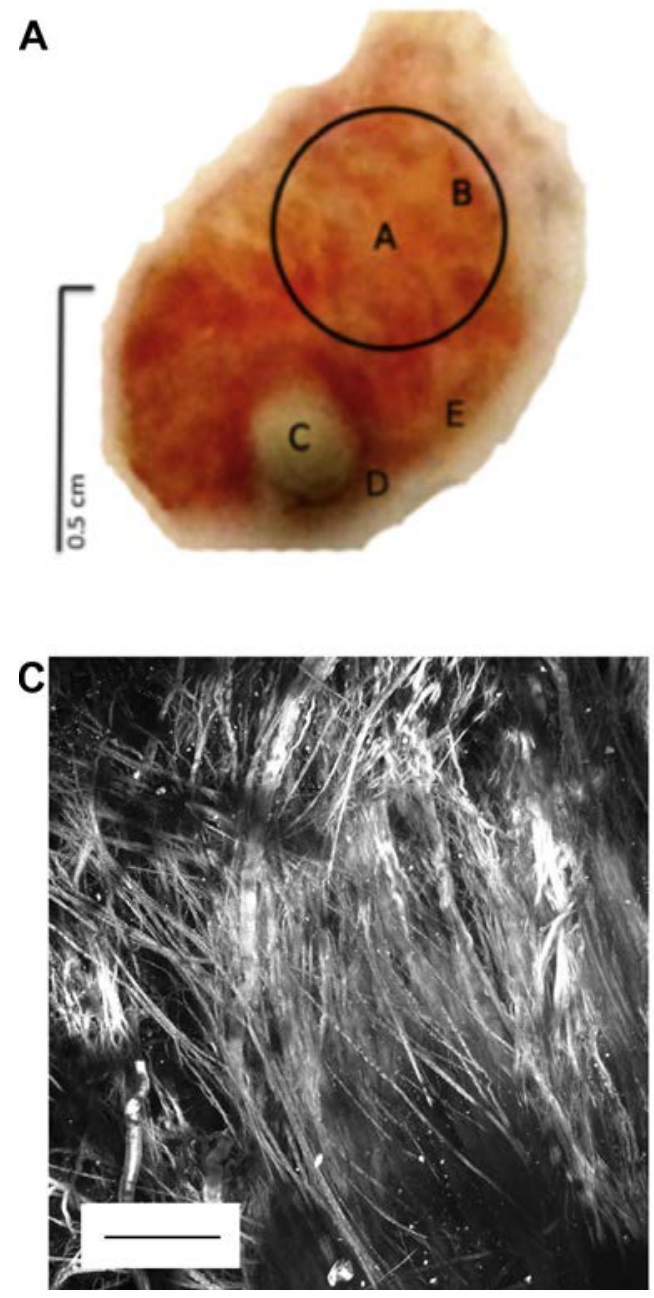

Figure 4. (A) Photograph of a ruptured aneurysm sample, luminal side. (B) Cartography of Young's modulus of the same sample, expressed by a color-coded map. Young's modulus is lowest at the blue zones and highest at the red ones, which correspond to the most rigid part of the aneurysm. Black circle

Neither compression (i.e., indentation test) nor tension loading (i.e., tensile test) are physiologic, also because the sample is not tested in its natural milieu. In addition, both tests give a relative measure of mechanical behavior that depends on the conditions of the test. Nonetheless, an indentation test might be better suited to study the local biomechanical properties of the aneurysm wall than a tensile test, because the former allows an estimation of biomechanical properties with a higher spatial resolution. Its importance is illustrated by the alternating zones of high and low elastic capacity, which were found by the indentation test and concords with the heterogeneity of aneurysm walls observed during surgery, with stiff, sometimes calcified, areas intermingled with pulsatile, often thin-walled zones. ${ }^{I 4}$ In addition, we observed a higher elastic capacity of UIAs compared with the unruptured zones of RIAs. We speculate that an increased stiffness in
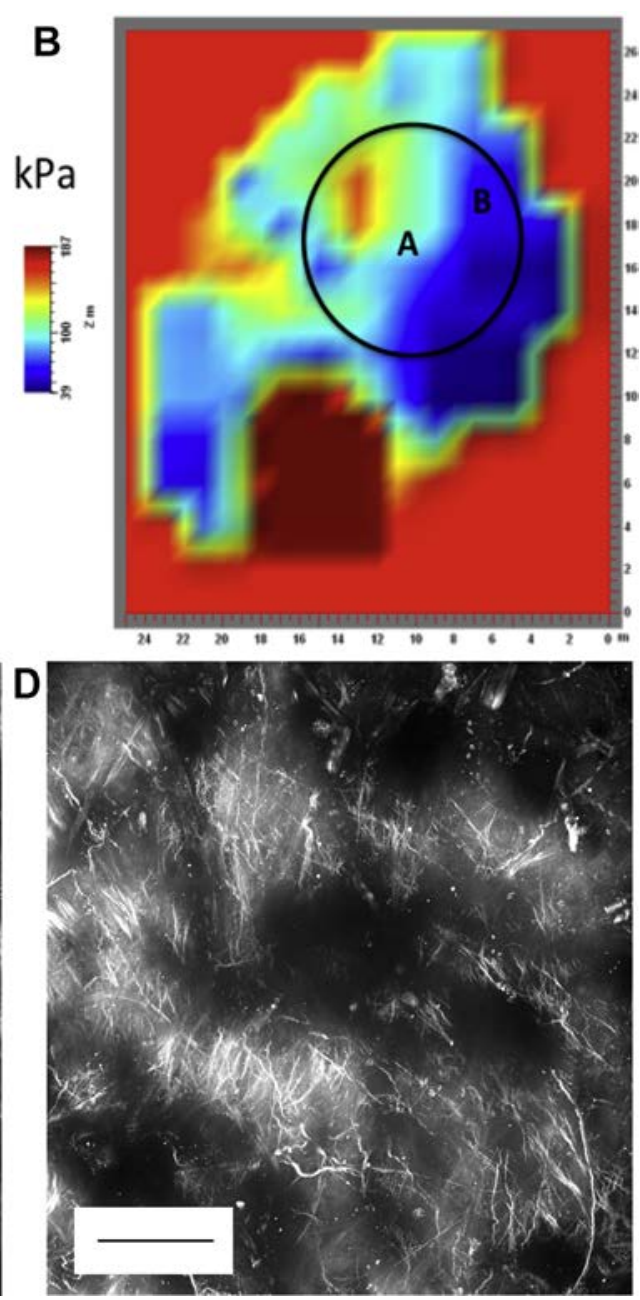

indicates the aneurysm fundus; $A$ marks its geometric center; $B$ indicates the rupture zone; $C$ indicates a calcification adjacent to the neck, comprised between $D$ and $E$. Two-photon microscopy of the same aneurysm sample at the unruptured $(\mathbf{C})$ and ruptured (D) zone, luminal side. Scale bar, $50 \mu \mathrm{m}$. $\mathrm{kPa}$, kilopascal.

nonruptured areas of RIAs could promote a local loss of elastic capacity by creep relaxation in a restricted area where hemodynamic stress is transmitted without the cushioning effect of the rest of the aneurysm. ${ }^{26}$ Thus, according to our results, it seems that decreasing of the Young's modulus takes place in a restricted area of the aneurysm where rupture occurs. Our results are in line with computational fluid dynamics findings that confirm the heterogeneity of wall shear stress on aneurysm wall. $^{12,26,27}$ These investigators ${ }^{12,26}$ found lower wall shear stress values at the rupture zone compared with the rest of the aneurysm wall, with a positive correlation between low wall shear stress and reduced thickness of the aneurysm wall. Based on this positive correlation, they ${ }^{12,26}$ hypothesized that wall shear stress reduction may cause depletion of endothelial cells and fragmentation of external elastic membrane and media, through the expression of 
endothelin-I and nitric oxide synthase. Fragmentation, rarefaction, and loss of network organization of external elastic lamina collagen fibers were observed at the rupture zone by microscopy. This finding provides a link between ultrastructural alterations and modifications of the biomechanical properties at the rupture zone.

The main limitations of the study are the selection bias (aneurysms for whom surgery was preferred to endovascular treatment, specimens of $<2 \mathrm{~mm}^{2}$, specimens examined within I8 hours of sampling) and that the results are not directly applicable to clinical practice because they come from an after treatment analysis. However, the study provides new insights into aneurysm biomechanical properties that could be used in the future as end-points for the assessment of UIA rupture risk. Phasecontrast magnetic resonance imaging-based elastography allows for the noninvasive evaluation of Young's modulus of human arteries. ${ }^{28}$ This magnetic resonance imaging technique could be applied to the noninvasive evaluation of biomechanical properties of aneurysm wall after validation by the indentation technique as soon as phase-contrast magnetic resonance imaging-based elastography spatial resolution is improved. ${ }^{28}$

\section{CONCLUSIONS}

The original application of the indentation technique of fresh aneurysmal wall samples found increased elastic capacity in the rupture zone and increased stiffness in the rest of the aneurysm. This finding could be the basis of further research aimed at building a biomechanical-based model of aneurysm rupture risk.

\section{REFERENCES}

I. UCAS Japan Investigators, Morita A, Kirino T, Hashi K, Aoki N, Fukuhara S, et al. The natural course of unruptured cerebral aneurysms in a Japanese cohort. N Engl J Med. 2012;366:2474-2482.

2. Wiebers DO, Whisnant JP, Huston J, Meissner I, Brown RD, Piepgras DG, et al. Unruptured intracranial aneurysms: natural history, clinical outcome, and risks of surgical and endovascular treatment. Lancet (Lond Engl). 2003;362:I03-IIO.

3. Juvela S, Poussa K, Lehto H, Porras M. Natural history of unruptured intracranial aneurysms: a longterm follow-up study. Stroke. 2013;44:24I4-242I.

4. Boussel L, Rayz V, McCulloch C, Martin A, Acevedo-Bolton G, Lawton M, et al. Aneurysm growth occurs at region of low wall shear stress: patientspecific correlation of hemodynamics and growth in a longitudinal study. Stroke. 2008;39:2997-3002.

5. Greving JP, Wermer MJH, Brown RD, Morita A, Juvela S, Yonekura M, et al. Development of the PHASES score for prediction of risk of rupture of intracranial aneurysms: a pooled analysis of six prospective cohort studies. Lancet Neurol. 2014;13:59-66.

6. Etminan N, Brown RD, Beseoglu K, Juvela S, Raymond J, Morita A, et al. The unruptured intracranial aneurysm treatment score: a multidisciplinary consensus. Neurology. 2015;85:88I-889.

7. Etminan N, Rinkel GJE. Cerebral aneurysms: cerebral aneurysm guidelines-more guidance needed. Nat Rev Neurol. 2015;II:490-49I.

8. Thompson BG, Brown RD, Amin-Hanjani S, Broderick J, Cockroft K, Connolly E, et al. Guidelines for the management of patients with unruptured intracranial aneurysms: A Guideline for Healthcare Professionals from the American Heart Association/American Stroke Association. Stroke. 2015;46:2368-2400.

9. MacDonald DJ, Finlay HM, Canham PB. Directional wall strength in saccular brain aneurysms from polarized light microscopy. Ann Biomed Eng. 2000;28:533-542.

Io. Costalat V, Sanchez M, Ambard D, Thines L, Lonjon N, Nicoud F, et al. Biomechanical wall properties of human intracranial aneurysms resected following surgical clipping (IRRAs Project). J Biomech. 20II;44:2685-269I.

II. Shojima M, Oshima M, Takagi K, Torii R, Hayakawa M, Katada K, et al. Magnitude and role of wall shear stress on cerebral aneurysm. Stroke. 2004;35:2500-2505.

I2. Xiang J, Natarajan SK, Tremmel M, Ma D, Mocco J, Hopkins LN, et al. Hemodynamicmorphologic discriminants for intracranial aneurysm rupture. Stroke. 20II;42:I44-I52.

I3. Robertson AM, Duan X, Aziz KM, Hill MR, Watkins SC, Cebral JR. Diversity in the Strength and structure of unruptured cerebral aneurysms. Ann Biomed Eng. 2015;43:1502-I515.

I4. Ishikawa T, Nakayama N, Yoshimoto T, Aoki T, Teresaka S, Nomura M, et al. How does spontaneous hemostasis occur in ruptured cerebral aneurysms? Preliminary investigation on 247 clipping surgeries. Surg Neurol. 2006;66:269-275 [discussion: 275-276].

I5. Masson I, Fialaire-Legendre A, Godin C, Boutouyrie P, Bierling P, Zidi M. Mechanical properties of arteries cryopreserved at $-80^{\circ} \mathrm{C}$ and -I50 ${ }^{\circ}$ C. Med Eng Phys. 2009;3I:825-832.

I6. Pailler-Mattei C, Bec S, Zahouani H. In vivo measurements of the elastic mechanical properties of human skin by indentation tests. Med Eng Phys. 2008;30:599-6o6.

17. Pailler-Mattéi C, Zahouani H. Analysis of adhesive behaviour of human skin in vivo by an indentation test. Tribol Int. 2006;39:I2-2I.

I8. Pailler-Mattéi C, Zahouani H. Study of adhesion forces and mechanical properties of human skin in vivo. J Adhes Sci Technol. 2004;18:1739-1758.

I9. Sneddon IN. The relation between load and penetration in the axisymmetric boussinesq problem for a punch of arbitrary profile. Int J Eng Sci. 1965;3:47-57.

20. Loubet JL, Georges JM, Marchesini O, Meille G. Vickers indentation curves of magnesium oxide (MgO). J Tribol. I984;106:43-48.

2I. Oliver WC, Pharr GM. An improved technique for determining hardness and elastic modulus using load and displacement sensing indentation experiments. J Mater Res. I992;7:I564-I583.

22. Denk W, Strickler JH, Webb WW. Two-photon laser scanning fluorescence microscopy. Science. I990;248:73-76.

23. Toth BK, Nasztanovics F, Bojtar I. Laboratory tests for strength paramaters of brain aneurysms. Acta Bioeng Biomech. 2007;9:3-7.

24. Steiger HJ, Aaslid R, Keller S, Reulen H-J. Strength, elasticity and viscoelastic properties of cerebral aneurysms. Heart Vessels. I989;5:4I-46.

25. Sanchez M. Identification Du Risque Individuel de Rupture Des Anévrysmes Cérébraux Intra Crâniens: Une Approche Biomécanicienne. Montpellier. 20I2;2: I-8.

26. Kadasi LM, Dent WC, Malek AM. Colocalization of thin-walled dome regions with low hemodynamic wall shear stress in unruptured cerebral aneurysms. J Neurosurg. 2013;II9:I72-I79.

27. Alfano JM, Kolega J, Natarajan SK, Xiang J, Paluch RA, Levy E, et al. Intracranial aneurysms occur more frequently at bifurcation sites that typically experience higher hemodynamic stresses. Neurosurgery. 2013;73:497-505.

28. Taviani V, Sutcliffe MPF, Wong P, Li ZY, Young V, Graves MJ, et al. In vivo non-invasive high resolution MR-based method for the determination of the elastic modulus of arterial vessels. Conf Proc IEEE Eng Med Biol Soc. 2008;2008:5569-5572.

Conflict of interest statement: The authors declare that the article content was composed in the absence of any commercial or financial relationships that could be construed as a potential conflict of interest.

Presented in part in abstract and oral presentation form at the 17th European Congress of Neurosurgery, plenary session, October 1-5, 2017, Venice, Italy. 